\title{
Modelling the effects of operating conditions on die melt temperature homogeneity in single screw extrusion
}

Link to publication record in Manchester Research Explorer

\section{Citation for published version (APA):}

Abeykoon, C., Li, K., McAfee, M., Martin, P. J., Deng, J., \& Kelly, A. L. (2010). Modelling the effects of operating conditions on die melt temperature homogeneity in single screw extrusion. In host publication (pp. 42-47)

\section{Published in:}

host publication

\section{Citing this paper}

Please note that where the full-text provided on Manchester Research Explorer is the Author Accepted Manuscript or Proof version this may differ from the final Published version. If citing, it is advised that you check and use the publisher's definitive version.

\section{General rights}

Copyright and moral rights for the publications made accessible in the Research Explorer are retained by the authors and/or other copyright owners and it is a condition of accessing publications that users recognise and abide by the legal requirements associated with these rights.

\section{Takedown policy}

If you believe that this document breaches copyright please refer to the University of Manchester's Takedown Procedures [http://man.ac.uk/04Y6Bo] or contact uml.scholarlycommunications@manchester.ac.uk providing relevant details, so we can investigate your claim.

\section{OPEN ACCESS}




\title{
Modelling the Effects of Operating Conditions on Die Melt Temperature Homogeneity in Single Screw Extrusion
}

\author{
Chamil Abeykoon*, Kang Li $^{* *}$, Marion McAfee ${ }^{* * *}$ \\ Peter J. Martin*, Jing Deng**, Adrian L. Kelly**** \\ *School of Mechanical and Aerospace Engineering, Queen's University \\ Belfast, Belfast, BT9 5AH, UK (Tel: +442890974236; e-mail: \\ yabeykoon01@qub.ac.uk,p.j.martin@qub.ac.uk) \\ ${ }^{* *}$ School of Electronics, Electrical Engineering and Computer Science, \\ Queen's University Belfast, Belfast, BT9 5AH, UK \\ (e-mail:k.li@qub.ac.uk,jdeng01@qub.ac.uk) \\ *** Department of Mechanical and Electronic Engineering, Institute of \\ Technology Sligo, Sligo, Ireland (e-mail: mcafee.marion@itsligo.ie) \\ **** IRC in Polymer Science and Technology, School of Engineering, \\ Design and Technology, University of Bradford, Bradford, BD7 1DP, \\ UK (e-mail: A.L.Kelly@bradford.ac.uk)
}

\begin{abstract}
The extrusion process is fundamental to the production of the vast majority of polymer and composite products in the plastics industry. The delivery of a melt which is homogenous in composition and temperature is paramount for achieving high quality extruded products. However, melting stability can be difficult to monitor/control via typical thermocouple measurements as they provide only a single point temperature measurement of melt flow. Previous studies have shown that die melt temperature changes considerably across the die and point based measurements are not sufficient to determine thermal homogeneity. In this work, the die melt temperature profile is monitored by a thermocouple mesh technique and the data obtained is used to formulate linear/nonlinear models to predict the effects of process settings on the die melt temperature homogeneity in a single screw extruder. A static nonlinear polynomial model is developed, which shows a good agreement with the measured data and provides a straight forward approach for investigating the effects of individual processing parameters on the melt flow homogeneity.
\end{abstract}

Keywords: Single Screw Extrusion, Melt Temperature Homogeneity, Thermocouple Mesh, Process Settings, Modelling.

\section{INTRODUCTION}

Achieving good thermal stability is a major requirement of the extrusion process in order to form high quality products. Previous studies (Kelly et al., 2006; Abeykoon et al., 2010; Rasid and Wood, 2003) have revealed that the thermal homogeneity of the melt is considerably affected by the process settings and that the melt flow temperature in the die changes according to its radial position. Therefore, use of the entire melt temperature profile as a measure of the process thermal stability is much more appropriate to ensure high quality products than a single point measurement. Although melt flow homogeneity may be improved slightly by introducing a filter or screen pack (gives better mixing), for better control, process settings should be appropriately optimised. Unfortunately, it is quite difficult to monitor the die temperature profile within a production environment and most extruders are instrumented only with conventional wall mounted thermocouples for thermal measurements. These are highly affected by the barrel wall temperature and they are not capable of measuring a melt temperature profile, or of detecting rapid variations in melt temperature (Schoppner et al., 2008).

Some thermal measurement methods (e.g. the thermocouple mesh and the fluorescence technique) may be used to measure melt temperature profiles. A grid of thermocouple wires which is used to observe the radial extrusion temperature profile is referred to as a thermocouple mesh. An emf is developed at each grid junction which can be correlated with the local melt temperature (Brown et al., 2004). The fluorescence technique involves doping the polymers with a temperature sensitive dye. Temperature is derived from changes in the fluorescence spectrum (Bur and Roth, 2004). However, these techniques are less suitable for use in a production environment due to constraints such as their complexity, limited durability, access requirements, disruptive effects on melt flow and output, the need to dope the polymer with additives etc. In fact, these techniques can be used to gather valuable process information in a research setting.

Moreover, understanding of the relationships between the 
process thermal stability and the adjustable process parameters are required to maintain the thermal conditions within tolerable limits. Relevant previous work in this field is discussed in the following sections.

\subsection{Effects of process settings on die melt temperature homogeneity}

Previous work by the authors (Abeykoon et al., 2010) discussed the effects of screw speed and barrel temperatures on the die melt temperature profile. A thermocouple mesh was used to measure the temperature profile of the melt flow prior to die entry. It was found that temperature variations increased as screw speed increased and the highest fluctuations existed a few millimetres away from the die wall, particularly at high screw speeds. Barrel temperature changes were shown to have a slight effect on the melt temperature profile.

Rasid and Wood (2003) used a thermocouple mesh inbetween the screw tip and die to measure the melt flow temperature profile with a low density polyethylene. They state that the metering section temperature had the highest influence on the melt temperature while the compression zone and die temperatures had negligible effects. Crabtree et al. (2008) argues that the extrudate temperature can be easily controlled by changing the metering zone temperature. However, this work was based on a conventional thermocouple measurements instead of measurements of the melt temperature profile.

Kelly et al. (2005, 2006, 2008) used a thermocouple mesh to monitor the melt temperature profile and presented a number of comprehensive studies on factors influencing the melt temperature homogeneity other than the process settings. Extruder screw geometry and polymer properties were also found to be as the significant parameters.

Bur et al. (2004) used a non invasive fluorescent measurement technique to measure the vertical temperature profile across the melt flow inside the extruder barrel (i.e. screw root to barrel wall) and discussed the effects of screw speed, screw geometry, and material specific behaviour on the melt temperature profile. Moreover, Bur and Roth (2004) used a fluorescent technique to measure a radial temperature profile in a reactive extrusion process.

\subsection{Models to predict melt temperature}

While attempts have been made to estimate melt temperature profile from first principles of mass, momentum and heat transfer, by finite difference methods (e.g. Tadmor et al. (1974)), these have limited accuracy in practice. Furthermore such models cannot be solved in real-time for process optimisation and control. Other workers have examined empirical system identification approaches to develop static models (e.g. Fontaine (1975); Hassan and Parnaby (1981); Germsuka et al. (1984); Chan and Lee (1984); Chan et al. (1986); Nelson et al. (1986)) and dynamic models (e.g. Kochhar and Parnaby (1977); Reber et al. (1973); Lin and Lee (1997); Tan et al. (2004)) to predict an average bulk melt temperature only. Furthermore, most of the existing thermal models are only representing linear relationships and are not be sufficient to represent the nonlinear processing behaviour over a wide operating range.

In this work, a thermocouple mesh technique (Brown et al., 2004) is used to measure the radial temperature profile across the die melt flow under different operating conditions and an attempt is made to model the effects of process settings on melt temperature profile at the die. The main objective is to explore an accurate and industrially compatible method to maintain the melt flow homogeneity rather than using less accurate conventional thermocouples. As outlined, a thermocouple mesh is not suitable in a production environment due to less fragility. However, the experimental information obtained can be used to formulate accurate models to predict the melt temperature profile based on the specified melt flow positions temperatures. Later, these models can be used in conjunction with a industrially compatible sensor for process control purposes which will be considered as a part of this project. The study reported in this paper is only focused on the melt temperature of a single screw extruder with one processing material.

\section{EQUIPMENT \& PROCEDURE}

All measurements were carried out on a $63.5 \mathrm{~mm}$ diameter (D) single screw extruder (Davis Standard BC-60). A tapered compression screw with $3: 1$ compression ratio (Feed-4D, Compression (or Melting)-10D, Metering 10D) was used to process the polymer material. The extruder was fitted with an adaptor $(\mathrm{D}=38 \mathrm{~mm})$ prior to a short cylindrical die with a $12 \mathrm{~mm}$ bore. The barrel has four separate temperature zones equipped with Davis Standard Dual Therm controllers.

Melt temperature profiles at the die were measured using a thermocouple mesh placed inbetween the adapter and the die as shown in Figure 1. A previous study (Kelly

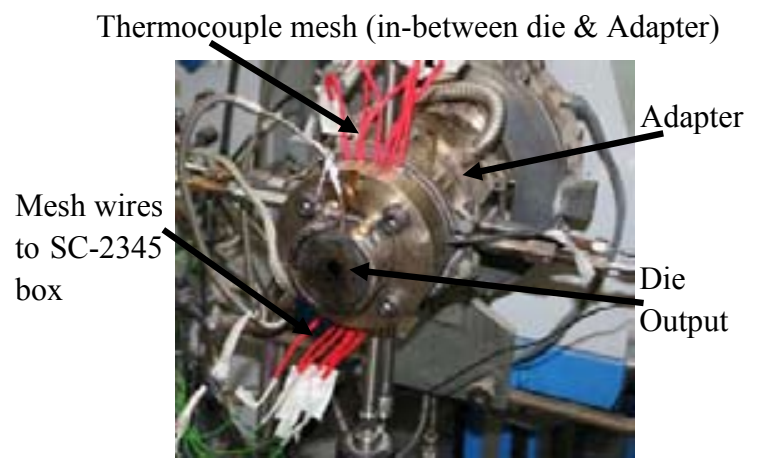

Fig. 1. Extruder die, adapter, and thermocouple mesh

et al., 2006) had confirmed that the die temperature measurements are symmetrical across the thermocouple mesh centreline. Therefore, five thermocouple wires were placed asymmetrically across the die melt flow to generate junctions along the diameter of the mesh and this asymmetric placement of wires gives the opportunity to increase the number of temperature measurements resolution across the melt flow. Finally, the melt temperatures measured at five points across the die melt flow were mirrored over the die centreline to obtain the complete die temperature profile. The die wall set temperature was used as the melt 
temperature at the $\pm 19 \mathrm{~mm}$ radial positions. Then, the final temperature profile was obtained by the 11 radial positions (distances from the die centre line to each radial position: $0 \mathrm{~mm}, \pm 3 \mathrm{~mm}, \pm 5 \mathrm{~mm}, \pm 8.5 \mathrm{~mm}, \pm 15 \mathrm{~mm}$, and $\pm 19 \mathrm{~mm}$ ) across the melt flow as illustrated in Figure 2 .

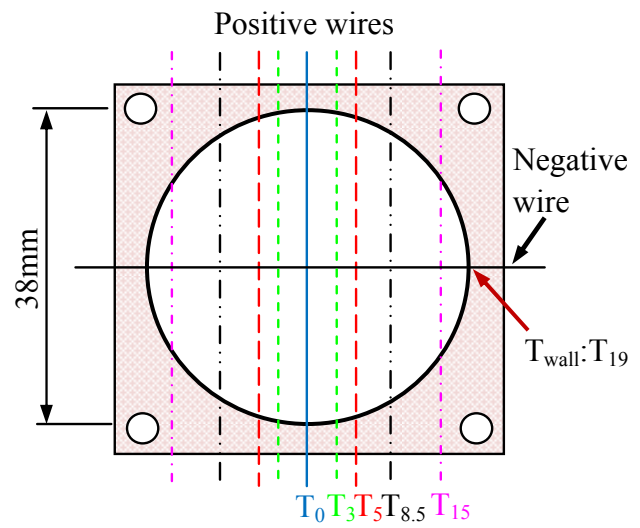

Fig. 2. The thermocouple mesh arrangement

A LabVIEW software program was developed to communicate between the experimental instruments and a PC. All thermocouple mesh temperature signals were acquired using a 16-bit DAQ card (National Instruments PCMCIA $6036 \mathrm{E})$ through a SC-2345 connector box.

The experimental trials were carried out on a recycled extrusion grade black high density polyethylene (HDPE), (MFI $0.16 \mathrm{~g} / 10 \mathrm{~min}$, density $0.967 \mathrm{~g} / \mathrm{cm}^{3}$, and $\sim 2.5 \%$ carbon black) provided by Cherry Pipes Ltd. The melt flow index (MFI) value is presented according to the ISO 1133 standard $\left(190^{\circ} \mathrm{C}, 2.16 \mathrm{~kg}\right)$. The extruder temperature settings were fixed as described in Table 1 and three experimental trials were carried out and named as: A (low temperature), B (medium temperature), and C (high temperature). The screw speed was adjusted from 10rpm

Table 1. Extruder barrel temperature settings

\begin{tabular}{|c|c|c|c|c|c|c|c|}
\hline \multirow{3}{*}{ Test } & \multicolumn{7}{|c|}{ Temperature settings $/{ }^{\circ} \mathrm{C}$} \\
\hline & \multicolumn{4}{|c|}{ Barrel Zones } & \multirow{2}{*}{ Clamp Ring } & \multirow{2}{*}{ Adapter } & \multirow{2}{*}{ Die } \\
\hline & 1 & 2 & 3 & 4 & & & \\
\hline $\mathrm{A}$ & 130 & 155 & 170 & 180 & 180 & 180 & 180 \\
\hline B & 140 & 170 & 185 & 200 & 200 & 200 & 200 \\
\hline $\mathrm{C}$ & 150 & 185 & 200 & 220 & 220 & 220 & 220 \\
\hline
\end{tabular}

to $90 \mathrm{rpm}$ in step sizes of 40rpm in tests $\mathrm{A}$ and $\mathrm{C}$ and in steps of $20 \mathrm{rpm}$ in test $\mathrm{B}$ with the extruder running for about nine minutes at each speed.

\section{MODELLING}

\subsection{System model identification}

The main aim is to model the effects of process settings on the shape of the die melt temperature profile (i.e. the variations of the melt temperature across the melt flow). Firstly the model inputs and outputs were identified. Six input parameters $(u 1-u 6)$ and one output parameter $(y 1)$ were considered for modelling as illustrated in Figure 3.

Inputs: screw speed $(N)$, die radial position $(R p)$, barrel set temperatures $\left(T_{b}\right)$ at each zone $\left(T_{1}, T_{2}, T_{3}, T_{4}\right)$. The set temperatures of the clamp ring, the adapter, and the die are always equal to $T_{4}$ in this study. If these values are different from $T_{4}$, it is possible to add them as three different model input parameters.

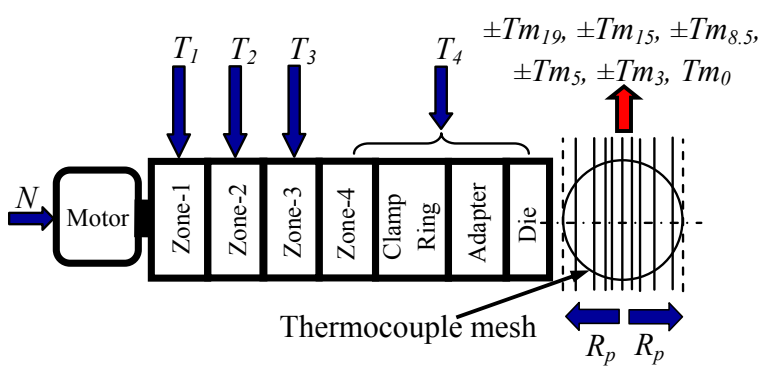

Fig. 3. Extruder model with selected inputs and output

Output: die melt temperature at each radial position

Shapes of the average die melt temperature profiles over last five minutes (4-9 minutes) at different screw speeds for tests $\mathrm{B}$ and $\mathrm{C}$ are shown in Figures 4 and 5 . The flatness

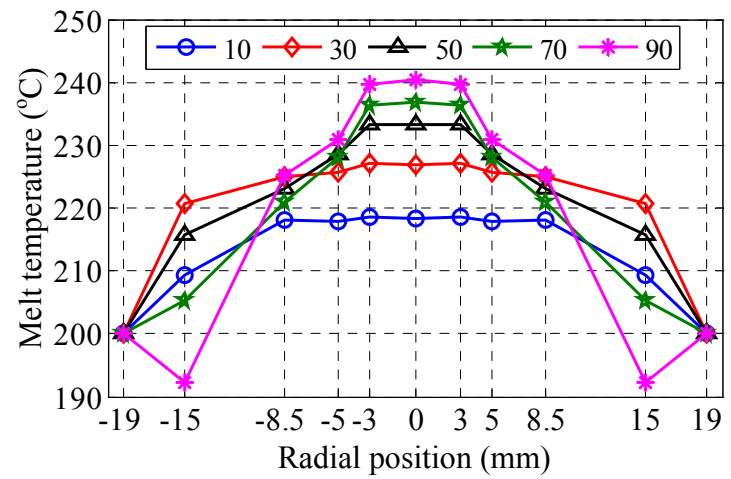

Fig. 4. Die temperature profiles from 10-90rpm:Test B

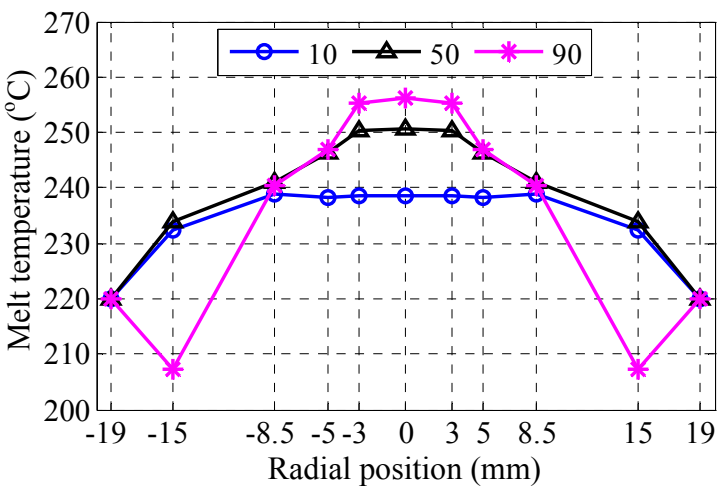

Fig. 5. Die temperature profiles from 10-90rpm:Test C

of the melt temperature profile decreases as the screw speed increases. The temperature profile is also affected by the barrel set temperatures as shown in Figures 4 and 5 . Melt temperature increases as the barrel set temperature increases.

\subsection{Model development and operation}

Each experimental trial was run over nine minutes at each set of process settings. Process signals were unsteady and contain transients within the first few minutes. Therefore, the data collected over the $7^{t h}$ and $8^{t h}$ minutes at 
the $1 \mathrm{~Hz}$ sampling rate were used for the model validation and development respectively. This is a steady state model which predicts the melt temperature values of the each radial position assigned by the radial position input. Eleven radial positions $\left(R_{p}\right.$ inputs: $0 \mathrm{~mm}, \pm 3 \mathrm{~mm}, \pm 8.5 \mathrm{~mm}$, $\pm 15 \mathrm{~mm}, \pm 19 \mathrm{~mm})$ predict a complete melt temperature profile across the die melt flow each second. The model estimates the melt temperature values of those eleven points individually by only changing the radial position input, while screw speed and barrel set temperatures remain constant. There are ten different processing situations with each having 660 input data points over one minute. Therefore each input signal contains 6600 data points. The model output contains the measured melt temperature at each radial position corresponding to the relevant inputs and the signal length is same as the input signal length.

The melt temperature profile at the die $\left(T_{p, \text { die }}\right)$ can be represented as a function of $N, R_{p}$, and $T_{b}$;

$$
T_{p, \text { die }}=f\left(N, R_{p}, T_{b}\right)
$$

This study is focused on developing a static model to predict the die temperature profile based on above relationship. Firstly, an attempt was made to identify a linear model to correlate the output with the inputs by approximating the function $f$. However, the linear model did not predict the temperature values accurately due to the significant nonlinearities in the process.

Secondly, a nonlinear polynomial model was adopted to approximate the function $f$. The predicted non-linear relationship is shown to give a reasonable agreement with the experimental data. Due to the strong nonlinearity of the polymer process, the maximum model power was selected as 6 . As a result, a large number of terms were included in the model, which may limit its practical application. However, only a few terms in this model were found to provide a significant contribution to the output. Sub-model selection algorithms, such as orthogonal least squares (OLS) (Chen et al., 1989) and fast recursive algorithm (FRA) (Li et al., 2005) can be applied to construct a parsimonious model with satisfactory generalisation capability. Due to the lower computation complexity and improved stability, an FRA was used as a sub-model selection algorithm here.

\subsection{Fast recursive algorithm (FRA)}

Suppose a nonlinear polynomial model can be expressed as:

$$
y=f\left(u_{1}, \cdots, u_{k}\right)
$$

where $y$ is the output, and $u_{i}$ are the system input variables. By using a polynomial function, this equation can be expressed as a linear-in-the-parameter model:

$$
y=\sum_{i=1}^{n} \theta_{i} \varphi_{i}(x)+e
$$

where $\varphi_{i}(\cdot), i=1, \cdots, n$ are all candidate model terms, and $x=\left[u_{1}, \cdots, u_{n}\right]^{T}$ is the model input vector.

Suppose N data samples are used for model training, then (3) can be re-written as

$$
\mathbf{y}=\Phi \Theta+\mathbf{e}
$$

where $\Phi=\left[\varphi_{1}, \cdots, \varphi_{n}\right] \in \Re^{N \times n}$ is the regression matrix, $\mathbf{y}=[y(1), \cdots, y(N)]^{T} \in \Re^{N}$ is the desired output, $\boldsymbol{\Theta}=$ $\left[\theta_{1}, \ldots, \theta_{n}\right]^{T} \in \Re^{n}$, and $\mathbf{e}=[e(1), \cdots, e(N)]^{T} \in \Re^{N}$ is the residual vector.

The well known least-square method solves the problem by minimising the cost function

$$
J(\Theta)=\mathbf{e}^{T} \mathbf{e}
$$

and the corresponding solution is given by

$$
\Theta=\left(\Phi^{\mathrm{T}} \Phi\right)^{-1} \Phi^{\mathrm{T}} \mathbf{y}
$$

However, due to the noise and correlation between regressors, the information matrix $\Phi^{\mathrm{T}} \Phi$ is always ill-conditioned in practice, which may lead to inaccurate calculation of the model coefficients $\Theta$. Ridge regression can prevent this problem, but gives a biased solution. Therefore, use of a subset selection algorithm eliminates this problem by selecting the most relevant and significant terms.

The fast recursive algorithm (FRA) utilised in this study can be presented by defining a recursive matrix $\left(M_{k}\right)$ and a residual matrix $\left(R_{k}\right)$ :

$$
\begin{aligned}
& \mathbf{M}_{\mathbf{k}} \triangleq \boldsymbol{\Phi}_{\mathbf{k}}^{\mathrm{T}} \boldsymbol{\Phi}_{\mathbf{k}} \quad k=1, \cdots, n \\
& \mathbf{R}_{\mathbf{k}} \triangleq I-\boldsymbol{\Phi}_{\mathbf{k}} \mathbf{M}_{\mathbf{k}}^{-\mathbf{1}} \boldsymbol{\Phi}_{\mathbf{k}}^{\mathrm{T}} \quad \mathbf{R}_{0} \triangleq I
\end{aligned}
$$

and the cost function in (5) can be rewritten as:

$$
J\left(\mathbf{P}_{k}\right)=\mathbf{y}^{\mathrm{T}} \mathbf{R}_{k} \mathbf{y}
$$

where $\mathbf{P}_{k}=\left[\mathbf{p}_{1}, \ldots, \mathbf{p}_{k}\right], k=1, \ldots, n$ and $p_{i}$ are selected model terms.

In this forward stepwise selection, polynomial terms are selected one-by-one based on their contribution to the final model. As shown in $\mathrm{Li}$ et al. (2005, 2006), if one more regressor $\varphi_{j}$ from the candidate term pool is to be selected, the net contribution of $\varphi_{j}$ to the cost function can be calculated as

$$
\begin{aligned}
\Delta J_{k+1}\left(\varphi_{j}\right) & =\mathbf{y}^{\mathrm{T}}\left(\mathbf{R}_{k+1}-\mathbf{R}_{k}\right) \mathbf{y} \\
& =\frac{\left(\mathbf{y}^{\mathrm{T}} \varphi_{j}^{(k)}\right)^{2}}{\varphi_{j}^{\mathrm{T}} \varphi_{j}^{(k)}}
\end{aligned}
$$

where $\varphi_{j}^{(k)} \triangleq \mathbf{R}_{k} \varphi_{j}, k+1 \leqslant j \leqslant m$.

The above net contribution can be further simplified by defining an auxiliary matrix $\mathbf{A} \in \Re^{k \times M}$ and a vector $\mathbf{a}_{\mathbf{y}} \in \Re^{M \times 1}$ with their elements given by

$$
\begin{gathered}
a_{i, j} \triangleq\left\{\begin{array}{l}
\left(p_{i}^{(i-1)}\right)^{\mathrm{T}} p_{j}, 1 \leq j \leq k \\
\left(p_{i}^{(i-1)}\right)^{\mathrm{T}} \varphi_{j}, k<j \leq M
\end{array}\right. \\
a_{i, y} \triangleq \begin{cases}\left(p_{i}^{(i-1)}\right)^{\mathrm{T}} \mathbf{y}, & 1 \leq i \leq k \\
\left(\varphi_{i}^{(k)}\right)^{\mathrm{T}} \mathbf{y}, & k<i \leq M\end{cases}
\end{gathered}
$$

According to the properties of $\mathbf{R}_{k}$ (Li et al., 2005, 2006), $a_{k, j}$ and $a_{k, y}$ can be updated recursively:

$$
\begin{gathered}
a_{k, j}=p_{k}^{\mathrm{T}} \varphi_{j}-\sum_{l=1}^{k-1} a_{l, k} a_{l, j} / a_{l, l} \\
k=1, \cdots, n, \quad j=1, \cdots, M . \\
a_{k, y}=p_{k}^{\mathrm{T}} \mathbf{y}-\sum_{l=1}^{k-1} a_{l, k} a_{l, y} / a_{l, l} \quad k=1, \cdots, n .
\end{gathered}
$$

Now, substituting (13) and (14) into (10), the net contribution of $\varphi_{j}, j=k+1, \cdots, N$ to the cost function can be expressed as: 


$$
\Delta J_{k+1}\left(\varphi_{j}\right)=\frac{a_{j, y}^{2}}{a_{j, j}}
$$

The model term that gives the largest contribution is then selected, and this procedure is continued until some criterion is met (e.g., Akaike's information criterion (AIC)(Nelles, 2001)) or a pre-set maximum number of centres are selected. To further reduce the calculation complexity in term selection, at the $(k+1)$-th step, $a_{j, j}^{(k+1)}$ and $a_{j, y}^{(k+1)}(j=k+1, \cdots, M)$ can be updated recursively instead of computing from (13) and (14):

$$
\begin{aligned}
& a_{j, j}^{(k+1)}=a_{j, j}^{(k)}-a_{k, j}^{2} /\left(a_{k, k}\right) \\
& a_{j, y}^{(k+1)}=a_{j, y}^{(k)}-a_{k, j} a_{k, y} /\left(a_{k, k}\right)
\end{aligned}
$$

At the end of each selection, these terms will be updated and stored for next comparison/selection. After a satisfactory model has been constructed, the model coefficients can now be computed recursively.

$$
\hat{\theta}_{j}=\left(a_{j, y}-\sum_{i=j+1}^{k} \hat{\theta}_{i} a_{j, i}\right) / a_{j, j}, j=k, k-1, \cdots, 1 .
$$

where the terms $a_{j, j}$ and $a_{j, y}$ in (18) are similar notations for the $a_{j, j}^{(k)}$ and $a_{j, y}^{(k)}$ terms used above. Finally, a more general model (i.e., terms and coefficients) is selected based on the defined process inputs and outputs from the sub model selection algorithm.

\section{DISCUSSION}

The linear static die melt temperature prediction model gave only about $30 \%$ fit with training data and could not be improved further. As the model performance was very poor, further details of the linear model are not presented in this paper.

For the nonlinear static die melt temperature prediction model $\left(T_{p, \text { die }}\right)$ selection, a number of different model combinations (i.e. with different orders and number of terms) were studied and the details of all of the models studied are shown in Table. 2 along with their root mean square errors (RMSE) with training data.

Table 2. Root mean square errors (with training data) of the studied nonlinear models with different orders and number of terms

\begin{tabular}{|c|c|c|c|c|c|}
\hline \multirow{2}{*}{\begin{tabular}{c} 
Number $\begin{array}{c}\text { of } \\
\text { terms }\end{array}$ \\
\cline { 2 - 6 }
\end{tabular}} & \multicolumn{5}{|c|}{$\begin{array}{c}\text { Root mean square error (RMSE) } \\
\text { with the selected order }\end{array}$} \\
\hline \hline 1 & 13.7 & 13.7 & 13.7 & 13.7 & 13.7 \\
\hline 2 & 7.05 & 7.05 & 7.05 & 7.05 & 7.05 \\
\hline 3 & 6.81 & 6.81 & 6.80 & 6.78 & 6.77 \\
\hline 4 & 5.93 & 5.68 & 5.67 & 5.72 & 5.78 \\
\hline 5 & 5.29 & 5.36 & 5.44 & 5.37 & 5.22 \\
\hline 6 & 5.23 & 5.28 & 4.95 & 3.95 & 3.77 \\
\hline 7 & 5.20 & 5.19 & 4.27 & 3.55 & 3.58 \\
\hline 8 & 5.18 & 5.12 & 3.74 & 3.18 & 3.23 \\
\hline 9 & 5.16 & 5.07 & 3.66 & $\mathbf{3 . 0 9}$ & 3.11 \\
\hline 10 & 5.15 & 5.06 & 3.64 & 3.02 & 3.05 \\
\hline 11 & 5.16 & 5.05 & 3.6 & 2.97 & 3.02 \\
\hline 12 & 5.15 & 5.05 & 3.52 & 2.93 & 2.95 \\
\hline
\end{tabular}

Finally a $6^{\text {th }}$ order model with 9 terms was selected as presented in equation (19) as further increase in the order or number of terms did not improve the model performance considerably.

$$
\begin{aligned}
T_{p, \text { die }}= & \left(1.267 * T_{2}\right) \quad-\left(1.378 e-04 * R_{P}^{2} * T_{4}\right) \\
& +\left(8.256 e-09 * N^{4} * R_{P}^{2}\right)+(0.346 * N) \\
& +\left(2.627 e-07 * N^{2} * R_{P}^{4}\right)-\left(1.516 e-05 * N * R_{P}^{4}\right) \\
& -\left(1.535 e-06 * N^{3} * R_{P}^{2}\right)+\left(2.413 e-03 * N * R_{P}^{2}\right) \\
& -\left(3.667 e-13 * N^{2} * T_{4}^{4}\right)
\end{aligned}
$$

The selected nonlinear model shows a $83.3 \%(\mathrm{RMSE}=3.09)$ fit with the training data and a $83.0 \%(\mathrm{RMSE}=3.12)$ fit with the test data. The measured and model estimated temperature profiles over two different screw speeds and barrel set temperatures with the nonlinear modelling error (measured-estimated) are shown in Figure 6.
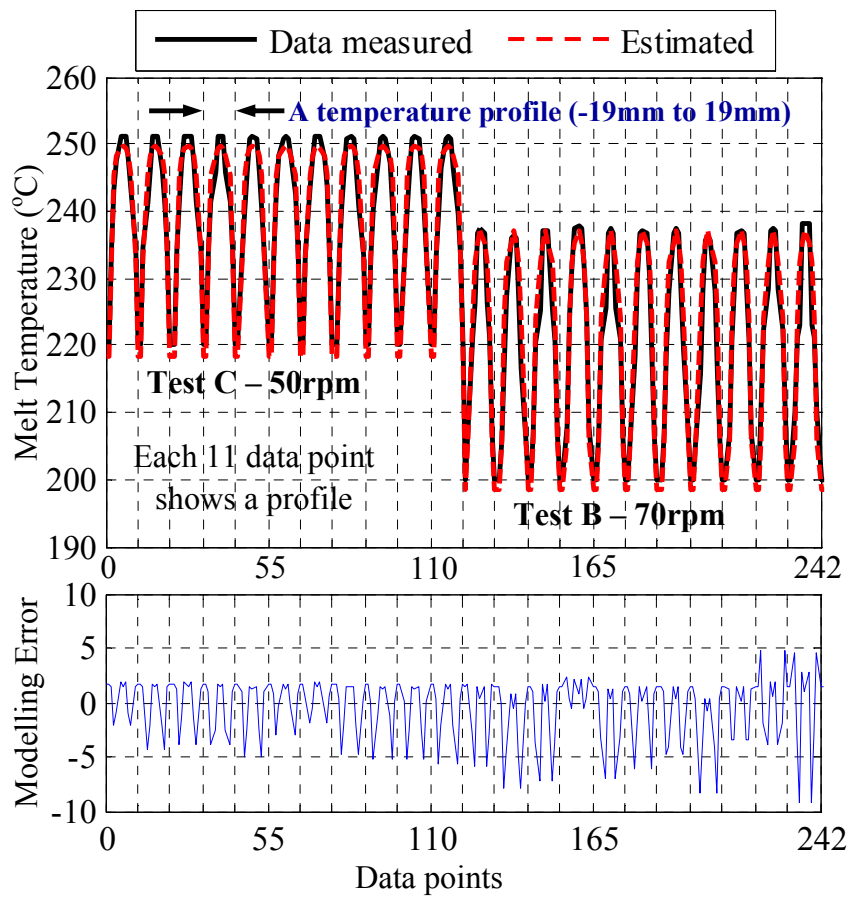

Fig. 6. Estimated and measured melt temperature profiles with modelling error for Test C: $50 \mathrm{rpm}$ (last 11s of the $\left.8^{\text {th }} \mathrm{min}\right)$ and Test B: $70 \mathrm{rpm}\left(1^{\text {st }} 11 \mathrm{~s}\right.$ of the $\left.8^{\text {th }} \mathrm{min}\right)$

Furthermore, each model term was closely examined to explore the effects of the individual processing parameters on the die melt temperature profile. The screw speed $(N)$ is identified as the most influential processing parameter. The melting zone temperature $\left(T_{2}\right)$ can be recognised as the most critical barrel zone temperature which affects the die temperature profile and hence the melt flow homogeneity. According to the previous findings of Rasid and Wood (2003) and Crabtree et al. (2008), screw speed is the most influential parameter on extruder output temperature while metering zone temperature $\left(T_{4}\right)$ is the most significant barrel zone temperature. However, according to the findings of this work metering zone temperature $\left(T_{4}\right)$ shows a less effect than the melting zone temperature $\left(T_{2}\right)$ on the shape of the die melt temperature profile while the effects of $T_{1}$ and $T_{3}$ are insignificant. $T_{1}$ is related to the feed zone which can be considered as negligible on the extruder melt output in this case. 
Indeed, melt temperature is not only a function of the process settings, consideration of the effects of material properties (e.g. thermal conductivity, melt consistency index, power law index, temperature coefficient of melt viscosity, shape/size/type of the resins) and machine geometry (e.g. channel depth, barrel diameter, surface conditions of barrel and screw, barrel thickness) may enhance the model capabilities to minimise the melt flow non-homogeneity, and this will be examined in future work.

\section{CONCLUSIONS}

A new static nonlinear polynomial model to predict the effects of process settings on the die melt flow homogeneity is presented. The model was used to identify the effects of individual processing parameter on the die melt flow homogeneity. Predictions from the proposed model are in agreement with previously reported experimental findings. In future work the model will be used to establish the optimum process settings to minimise melt flow nonhomogeneity. Moreover, this study is still in its initial stage and future consideration of the effects of material properties and machine geometrical parameters should help to improve the model performance further. In addition the development of a dynamic model is underway. This will help to demonstrate a potential method for determining melt flow homogeneity in-process and to buildup a control strategy (i.e. an industrial soft sensor) to obtain the required melt flow homogeneity in extrusion by manipulating the process settings. Moreover, a method to extrapolate the control strategy on other machine geometries and materials will also be examined.

\section{ACKNOWLEDGEMENTS}

This work was supported by the UK's Engineering and Physical Sciences Research Council (EPSRC) under grant number EP/F021070/1. The authors would like to thank J. Kissick, G. Garrett, S. Armstrong, and Dr. B. Millar of Queen's University Belfast and Dr. E. C. Brown, K. Howell, S. Brook, and Dr. Tim Gough from the University of Bradford, for their assistance. Also, the support provided by Cherry Pipes Ltd in providing materials is greatly acknowledged.

\section{REFERENCES}

Abeykoon, C., McAfee, M., Li, K., Kelly, A. L., Brown, E. C., 2010. Monitoring the effect of operating conditions on melt temperature homogeneity in single-screw extrusion. SPE-ANTEC Tech. papers, 1799-1806.

Brown, E. C., Kelly, A. L., Coates, P. D., 2004. Melt temperature field measurement in single screw extrusion using thermocouple meshes. Rev. Sci. Instrum. 75 (11), 4742-4748.

Bur, A. J., Roth, S. C., 2004. Fluorescence temperature measurements: Methodology for applications to process monitoring. Polym. Eng. Sci. 44 (5), 898-908.

Bur, A. J., Roth, S. C., Spalding, M. A., Baugh, D. W., Koppi, K. A., Buzanowski, W. C., 2004. Temperature gradients in the channels of a single-screw extruder. Polym. Eng. Sci. 44 (11), 2148-2157.

Chan, D., Lee, L. J., 1984. Dynamic modelling of a single screw plasticating extruder. SPE-ANTEC Tech. papers, 77-80.
Chan, D., Nelson, R. W., Lee, L. J., 1986. Dynamic behavior of a single screw plasticating extruder part II: dynamic modeling. Polym. Eng. Sci. 26 (2), 152-161.

Chen, S., Billings, S., Luo, W., 1989. Orthogonal least squares methods and their application to non-linear system identification. Int. J. Control 50, 1873-1896.

Crabtree, S. L., Spalding, M. A., Pavlicek, C. L., 2008. Single-screw extruder zone temperature selection for optimized performance. SPE-ANTEC Tech. papers 3, 1406-1411.

Fontaine, W., 1975. Ph.D. thesis, Ohio State University, Ohio.

Germsuka, D. D., Taylor, P. A., Wright, J. D., 1984. Adaptive and multivariable control of a single screw extrusion system. Can. J. Chem. Eng. 62 (6), 790-801.

Hassan, G. A., Parnaby, J., 1981. Model reference optimal steady-state adaptive computer control of plastics extrusion processes. Polym. Eng. Sci. 21 (5), 276-284.

Kelly, A. L., Brown, E. C., Coates, P. D., 2005. Melt temperature field measurement in single screw extrusion: influence of melt pressure and die geometry. $S P E$ ANTEC tech papers 1, 291-295.

Kelly, A. L., Brown, E. C., Coates, P. D., 2006. The effect of screw geometry on melt temperature profile in single screw extrusion. Polym. Eng. Sci. 46 (12), 1706-1714.

Kelly, A. L., Brown, E. C., Howell, K., Coates, P. D., 2008. Melt temperature field measurement in extrusion using thermocouple meshes. Plast. Rubb. Comp. 37, 151-157.

Kochhar, A. K., Parnaby, J., 1977. Dynamical modelling and control of plastics extrusion processes. Automatica 13 (2), 177-183.

Li, K., Peng, J., Bai, E., 2006. A two-stage algorithm for identification of nonlinear dynamic systems. Automatica 42 (7), 1189-1197.

Li, K., Peng, J., Irwin, G. W., 2005. A fast nonlinear model identification method. IEEE Trans. Autom. Control 50 (8), 1211-1216.

Lin, Y. J., Lee, G., 1997. System identification for state feedback integral observer control of polymer plastic extrusion. Polym. Plast. Technol. Eng. 36 (5), 749-775.

Nelles, O., 2001. Nonlinear System Identification. Berlin:Springer.

Nelson, R. W., Chan, D., Yang, B., Lee, L. J., 1986. Dynamic behavior of a single screw plasticating extruder part i: Experimental study. Polym. Eng. Sci. 26 (2), 144-151.

Rasid, R., Wood, A. K., 2003. Effect of process variables on melt tempearture profiles in extrusion process using single screw plastics extruder. Plast. Rubb. Comp. 32 (5), 193-198.

Reber, D. H., Lynn, R. E., Freeh, E. J., 1973. A mathematical model for predicting dynamic behavior of a plasticating extruder. Polym. Eng. Sci. 13 (5), 346-356.

Schoppner, V., Enns, K., Anger, K., Giese, E., 2008. Dynamic temperature measurement in polymer processing. SPE-ANTEC Tech. papers 1, 228-232.

Tadmor, Z., Lipshitz, S. D., Lavie, R., 1974. Dynamic model of a plasticating extruder. Polym. Eng. Sci. 14 (2), 112-119.

Tan, L. P., Lotfi, A., Lai, E., Hull, J. B., 2004. Soft computing applications in dynamic model identification of polymer extrusion process. Appl. Soft Comput. 4 (4), 345-355. 